\title{
Câncer cervical: uma análise descritiva da incidência, mortalidade e métodos de rastreamento em diferentes países
}

\author{
Cervical cancer: a descriptive essay of incidence, mortality and screening methods in different \\ countries
}

\author{
R. A. da Trindade* \\ Departamento de Análises Clínicas e Toxicológicas, Faculdade de Farmácia, Universidade Federal do Rio de Janeiro, \\ 21941-902, Rio de Janeiro-RJ, Brasil \\ *rtrindade@pharma.ufrj.br
}

(Recebido em 03 de abril de 2019; aceito em 23 de dezembro de 2019)

O câncer cervical permanece um desafio para as autoridades brasileiras de saúde pública, pois ainda é a terceira maior causa de mortes por câncer em mulheres, não obstante ser uma doença evitável e curável quando detectada precocemente. Baseado em dados epidemiológicos da Organização Mundial de Saúde e em pesquisa bibliográfica documental, esse texto traz uma descrição temporal da incidência e mortalidade do câncer cervical, além das principais características dos métodos de rastreamento populacional empregados no Brasil e em outros nove países, a saber: Estados Unidos da América, Canadá, Reino Unido, Alemanha, França, Itália, Japão, Holanda e Chile. O texto ressalta e discute as ações e estratégias que estão sendo implementadas nesses países para a redução das taxas de incidência e mortalidade por câncer cervical. Palavras-chave: Câncer de colo do útero, Programas de rastreamento, Serviços de saúde.

Cervical cancer remains as a challenge for the Brazilian public health authorities. This disease is the third leading cause of cancer deaths in women, although it is an avoidable and curable disease when early detected. Based on epidemiological data from the World Health Organization and specialized literatures, this essay describes the incidence and mortality of cervical cancer, and the main characteristics of population screening methods employed in Brazil and other nine countries: United States of America, Canada, United Kingdom, Germany, France, Italy, Japan, The Netherlands and Chile. The text emphasizes and discusses actions and strategies that have been implemented in such countries to reduce incidence and mortality rates for cervical cancer.

Keywords: Health services, Screening programs, Uterine cervical neoplasms.

\section{INTRODUÇÃO}

O câncer cervical permanece um desafio para as autoridades brasileiras de saúde pública. Segundo o Instituto Nacional do Câncer (INCA) [1], o câncer cervical foi a terceira maior causa de mortes por câncer em mulheres $(6,01 \%)$ no período de 2009-2014 no Brasil, situando-se abaixo somente dos cânceres de mama $(15,62 \%)$ e brônquios/pulmões $(10,51 \%)$. Para efeitos de comparação, em países como os Estados Unidos da América, Canadá, Reino Unido, França e Itália, o câncer cervical não consta nem mesmo entre os dez tipos de câncer com maior incidência ou causa de morte em mulheres [2].

Diferentemente do câncer de mama, que compreende causas multifatoriais incluindo fatores de risco não modificáveis [3], tais como idade, sexo, pré-disposição genética, entre outros, e o câncer de pulmão, cujo tratamento ainda apresenta limitações para a cura completa [4]. O câncer cervical, em sua maioria, está etiologicamente associado à infecção pelo HPV [5, 6], particularmente os subtipos de alto risco (hrHPV) 16 e 18, que possui evolução lenta e progressiva [7] e apresenta sinais precoces indicativos da sua evolução que podem ser detectados por exames citológicos não invasivos [8]. Portanto, apesar de grave, o câncer cervical pode ser evitado e, se detectado precocemente, tem elevada chance de cura e alta taxa de sobrevida [9].

Para fins de prevenção populacional, basicamente, há dois modelos de rastreamento: organizado (organised screening) e oportunístico (opportunistic ou spontaneous screening) [10]. O primeiro é aplicado a grupos específicos de indivíduos, delimitados pelo maior risco de desenvolver a doença ou por uma maior incidência. Esses grupos são alocados em programas governamentais de rastreamento, sob os quais, os indivíduos adquirem o direito de realizar testes ou exames que 
detectem sinais precoces da doença. Portanto, tal exame deve ser capaz de detectar a doença em seu estágio inicial, e sua aplicação, em termos de custo-benefício, deve ser cientificamente comprovada por estudos pilotos. Há situações em que o direito ao rastreamento é formalizado pelo envio sistemático de cartas-convites individualizadas, sendo assim uma busca ativa da populaçãoalvo. Além disso, o modelo organizado prevê protocolos bem definidos de tratamento e seguimento caso alguma anormalidade seja detectada no exame de rastreio, e preconiza o controle de toda a informação pertinente ao programa para subsidiar os indicadores epidemiológicos [11]. O segundo modelo, por outro lado, não envolve grupos delimitados, ainda que eles existam subjacentemente. No modelo oportunístico, como o nome sugere, nas oportunidades em que o indivíduo procura atendimento médico espontâneo é então ofertada ao indivíduo a possibilidade de realizar o exame ou teste de rastreamento. A existência ou não de um convite formal à realização do teste, ou alguma outra forma de busca ativa da população alvo, costuma ser o ponto de diferenciação entre os métodos que apresentam vários pontos de convergência [11].

O câncer cervical é uma doença para a qual, praticamente, todos os países possuem estratégias e/ou programas governamentais de rastreamento, adotando um dos dois modelos descritos acima ou mesmo os dois modelos. Cada país define seu programa por critérios próprios e de acordo com a conveniência social, política, disponibilidade financeira e os estudos epidemiológicos baseados em evidências de eficiência. Consequentemente, cada país também apresenta diferentes taxas de eficiências reveladas pelos indicadores de incidência e mortalidade [12]. No Brasil, embora seja oficialmente adotado o modelo populacional/organizado [13], na prática, em razão de não haver um sistema de busca ativa da população-alvo, por exemplo, por cartas-convites individuais, o que ocorre é o padrão oportunístico. Nesse caso, ao realizar uma consulta com clínico geral ou ginecologista, é ofertada à mulher a oportunidade de realizar o exame de Papanicolaou [14].

A introdução do exame de Papanicolaou a partir das décadas de 1940-1950, como forma de rastreamento preliminar das lesões celulares que podem evoluir para câncer, causou impactos significativos na incidência e mortalidade por câncer cervical em muitos países, particularmente, naqueles que adotaram o exame de forma regular e sistematizada. Entretanto, este fenômeno não ocorreu de forma tão impactante no Brasil. Portanto, a manutenção de taxas altas de incidência e mortalidade causada pelo câncer cervical no Brasil, sem mudanças significativas nos últimos anos, ainda que medidas governamentais e técnicas para a melhoria no rastreamento venham sendo implantadas, ensejou a escrita deste ensaio crítico-descritivo. Aqui, objetivou-se descrever sucintamente a incidência, a mortalidade e os métodos de rastreamento populacional com suas principais características em países com diferentes parâmetros socioeconômicos, relatando pontos que estimulem a reflexão sobre as razões pelas quais as estratégias empregadas no Brasil não estejam surtindo os efeitos benéficos esperados sobre as taxas epidemiológicas do câncer cervical.

\section{MATERIAL E MÉTODOS}

Em termos metodológicos, para delimitar a abrangência deste texto foram selecionados dez países, incluindo o Brasil, aplicando-se critérios econômicos, geográficos e sociais. O critério econômico se deu por países que tivessem uma economia forte e estável, sendo selecionados os países integrantes do bloco informal econômico conhecido por G7. Este grupo é constituído pelos países: Estados Unidos da América, Canadá, Reino Unido, Alemanha, França, Itália e Japão, que juntos detém aproximadamente 50\% do PIB (produto interno bruto) mundial [15]. O critério geográfico se deu pela inclusão de ao menos um país da América do Sul, além do Brasil. Para isso, selecionou-se o país com maior PIB per capita da região e o Chile foi o país incluído [16]. Como critério social, a Holanda foi selecionada por ter sido o país europeu com o maior índice de qualidade geral no sistema de cuidados da saúde (Health Care Index 81.08) no ano de 2018 [17], completando assim os dez países estudados neste ensaio: Brasil, Chile, Holanda, Estados Unidos da América, Canadá, Reino Unido, França, Itália, Japão e Alemanha.

Ainda para fins metodológicos, ressalta-se que esse ensaio consiste de uma pesquisa bibliográfica documental com análise secundária de dados primários. Os dados primários relativos à incidência e mortalidade foram obtidos nos sítios eletrônicos das agências internacionais de controle de informações epidemiológicas sobre saúde, doenças e câncer, a saber: Centers for Disease Control and Prevention (CDC), World Health Organization (WHO) e The International 
Agency for Research on Cancer (IARC) [2, 19, 20, 21]. As informações sobre os métodos de rastreamento utilizados nos diferentes países foram pesquisadas nos sítios eletrônicos das agências nacionais de saúde de cada país selecionado, além de documentos oficiais governamentais, tais como leis e decretos, que estabelecem e/ou regulamentam os programas de prevenção e tratamento nos respectivos países: Instituto Nacional de Câncer José Alencar Gomes da Silva (INCA/Br), Ministério de Salud del Chile (MINSAL/Cl), National Health Service of United Kington (NHS/UK), Nacional Institute for Public Health and the Environment (RIVM/Nl), L'Institut national du cancer (INCa/Fr), Public Health Agency of Canada (PHAC/Ca), American Cancer Society (ACS/EUA).

Para complementar as informações e aprofundamento da discussão dos dados obtidos, também foram incluídos artigos científicos publicados em periódicos especializados com relatos de casos e situações clínicas-epidemiológicas nesses países, bem como mudanças nas estratégias nos programas de rastreamento que foram ou estejam sendo implementadas para controle do câncer cervical. Todas as fontes bibliográficas consultadas, inclusive os sítios eletrônicos das agências governamentais dos países, foram listadas nas referências bibliográficas ao final do texto.

\section{RESULTADOS E DISCUSSÃO}

\subsection{Mortalidade por câncer cervical}

Segundo os dados da IARC de 2012, o câncer cervical foi o terceiro tipo de câncer mais incidente (Taxa ASR 14,0/100 mil mulheres; ASR: age-standardised rate ou taxa de mortalidade padronizada ou ajustada para idade) e a quarta maior causa (Taxa ASR 6,8/100 mil mulheres) de morte em mulheres no mundo [18]. Entretanto, a distribuição das taxas do câncer cervical é bastante variada nos diferentes continentes e, mais ainda, em diferentes países e estados, independentemente de possuir ou não algum programa governamental de prevenção. A Figura 1 mostra a evolução temporal das taxas de mortalidade [19] por câncer cervical nos dez países selecionado em dois períodos: o primeiro de 1950 a 2013 (Figura 1A) e o segundo de 1990 a 2013 (Figura 1B). Esse mostra um recorte temporal que ressalta o período em que os países haviam implantado ou estavam em fase de implantação de programas de rastreamento populacional.

Inicialmente, é importante salientar que a análise destes gráficos de dados temporais deve ser realizada com cautela, conforme recomendação da própria IARC, pois ocorrem mudanças nas metodologias de coleta e na formatação dos dados pelas agências internas de cada país, fazendo com que a comparação dos dados represente tão somente uma estimativa em termos de evolução temporal, mesmo que a priori, os dados tenham sido padronizados (ex.: incidência/mortalidade por 100 mil mulheres) e ajustados (ex.: incidência/mortalidade por faixa etária). Outro fato importante de ressaltar é que nos anos 50, o exame de Papanicolaou não era amplamente utilizado e poucos países o adotavam como exame diagnóstico preventivo. Além disso, os registros de óbitos por causas específicas também não eram completamente detalhados como são atualmente. Por isso, observam-se aumentos nas taxas de mortalidade entre os anos 1950 e 1975 em quase todos os países com exceção do Reino Unido e EUA. Isso provavelmente se deve à implantação de um registro descritivo mais detalhado da causa-morte específica quando decorrente do "câncer cervical", ao invés de uma descrição generalista de "câncer de útero". O Chile é um exemplo característico desse aumento na mortalidade por câncer cervical que se seguiu até o ano de 1975.

Segundo Arrossi et al. (2003) [22] concomitantemente a este aumento na mortalidade por câncer cervical no Chile, houve uma redução na mortalidade por câncer uterino. Essa informação corrobora a possível mudança na forma de registro desses óbitos, que passaram a conter informações mais específicas da causa-morte, que em contrapartida, aumentou em termos de registro quantitativo as causas de morte por câncer cervical. Portanto, os aumentos observados podem ser mais aparentes que reais, visto que ao longo desses anos os países passaram por transições epidemiológicas marcantes e também sistematizaram a coleta de dados em saúde pública. Convém ressaltar que para o Brasil, o registro sistematizado de mortes por câncer cervical iniciou somente a partir de 1979 e, portanto, os dados constam apenas a partir desse ano. 
A

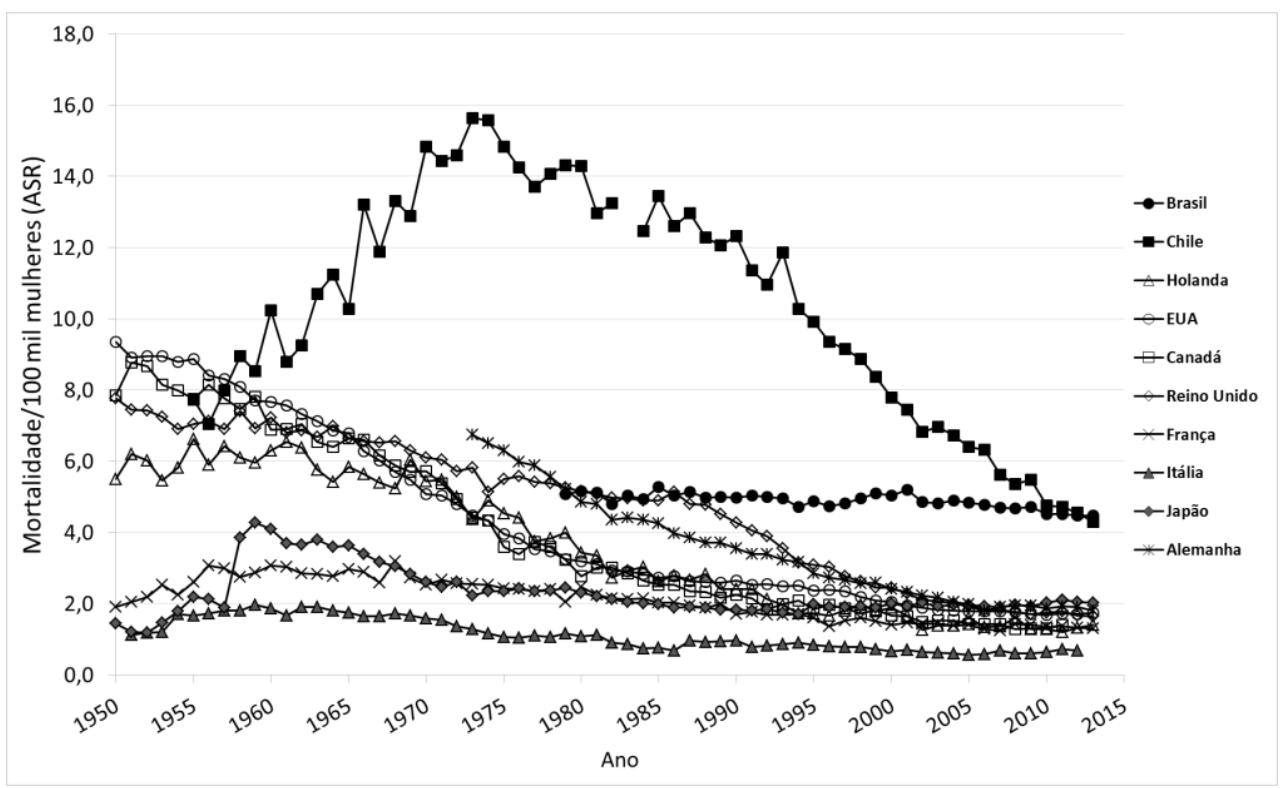

B

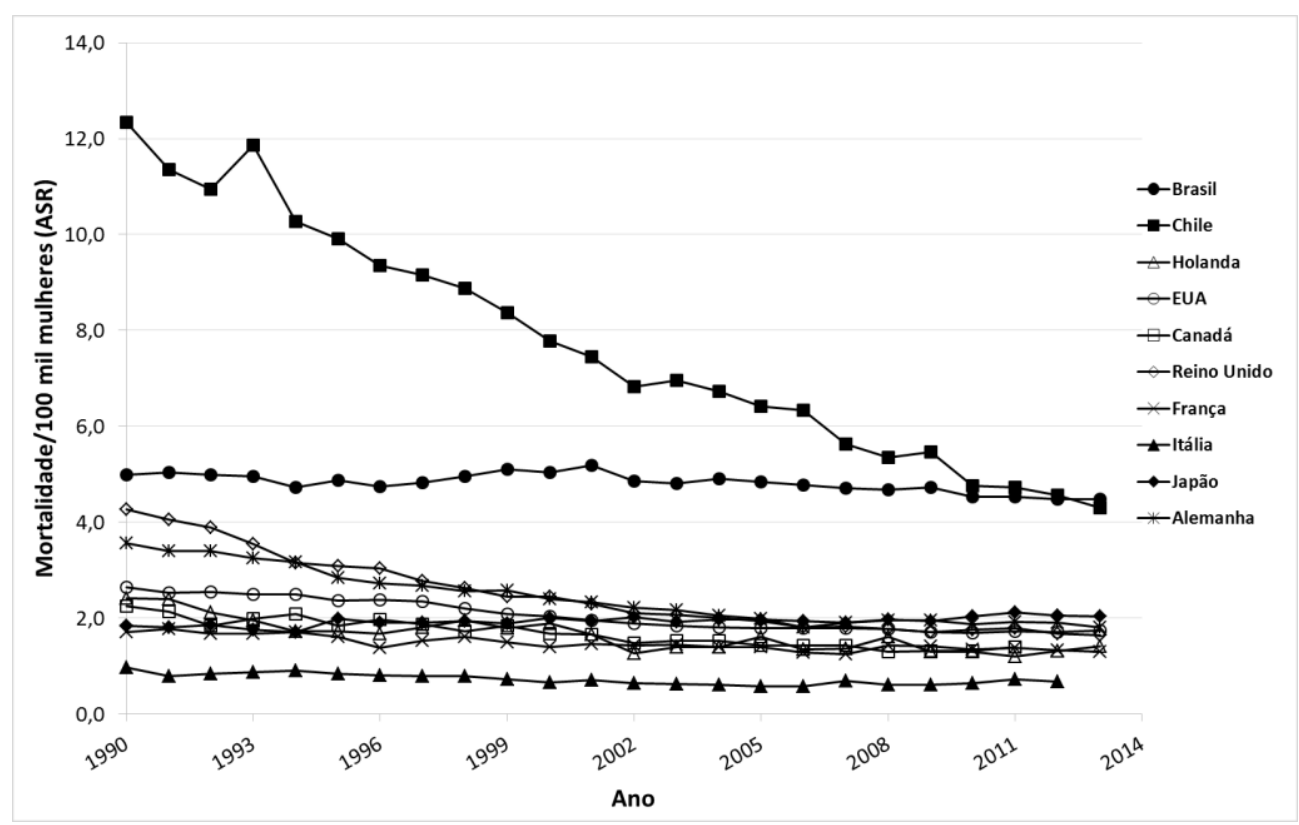

Figura 1. Evolução das taxas de mortalidade por câncer cervical nos dez países analisados entre os períodos de (A) 1950 a 2013 e (B) 1990 a 2013. Fonte primária dos dados: WHO, 2016 [19]. Disponível em <http://www-dep.iarc.fr/WHOdb/WHOdb.htm>. Acesso em 31 Ago 2017.

Considerando o exposto acima, a Figura 1B mostra o recorte da evolução temporal na mortalidade por câncer cervical a partir dos anos 1990. Nesse período, todos os países utilizavam o exame de Papanicolaou regularmente como método de diagnóstico, porém, apenas alguns possuíam programas regulares de rastreamento populacional, tais como o Canadá e o Japão, que iniciaram seus programas em 1960 e 1983, respectivamente. Outros ainda estavam em fase muito inicial da implantação de seus programas de rastreamento, tais como a Holanda, França, EUA e Itália que implantaram programas em 1989, 1990, 1991 e 1996, respectivamente [23]. Desta forma, esse recorte temporal reforça a constatação de como a implantação de programas regulares de rastreamento populacional influenciou as taxas de mortalidade por câncer cervical nos últimos anos. Observou-se que o Chile apresentou uma marcante e contínua redução na mortalidade por câncer cervical a partir do ano 1973, data em que o país apresentou pico na taxa de mortalidade $(15,64 / 100$ mil mulheres). Não foram observadas reduções expressivas nas mortalidades por câncer 
cervical no Japão, Canadá, França e Itália, porém, nesses países, as taxas observadas eram bastante baixas: $\pm 2,0, \pm 2,0,<2,0 \mathrm{e}<1,0 / 100$ mil mulheres, respectivamente. Reino Unido e Holanda também apresentaram reduções discretas em suas taxas de mortalidade. É interessante notar que a Alemanha tem apresentado taxas de mortalidade reduzidas a cada ano, ainda que não possua programa oficial de rastreamento nacional. Os EUA têm mantido suas taxas de mortalidade por câncer cervical abaixo de 2,0/100 mil mulheres desde os anos 2000. De forma geral, após a implantação de programas de rastreamento populacional, as taxas de mortalidade têm sido reduzidas ou mantidas em valores baixos nesses países. O Brasil é a exceção, pois não apresentou redução significativa nas suas taxas de mortalidade, mesmo possuindo um programa nacional de rastreamento, mantendo taxas entre 4,0 e 5,0/100 mil mulheres. Essas taxas são mais que o dobro das taxas encontradas nos EUA que, para fins de comparação, possui uma base populacional tão extensa quanto à brasileira.

Convém salientar que as taxas de mortalidade são indicadores que sofrem influências não apenas da eficiência do diagnóstico precoce, mas principalmente do seguimento/acompanhamento da paciente, tal como tempo de espera e tratamento adequado, tipo de lesão encontrada, enfim, de todo o funcionamento da rede integral de atendimento. Sendo assim, o uso do indicador 'incidência' pode se relacionar melhor com os métodos de prevenção e diagnóstico precoce empregados em cada país, principalmente em termos de câncer cervical que, de modo geral, apresenta uma evolução bastante lenta, agente etiológico bem definido e, portanto, sua ocorrência pode ser plenamente evitada na maioria dos casos.

\subsection{Incidência do câncer cervical}

Diferentemente da mortalidade, as taxas de incidências do câncer cervical não estão disponíveis para alguns países, principalmente nos períodos anteriores à implantação dos programas de rastreamento, que preconiza obrigatoriamente a obtenção desse indicador epidemiológico. $\mathrm{Na}$ busca dos dados epidemiológicos [20] realizada para escrita e atualização do presente artigo não foi encontrada informação anterior ao ano de 1998 sobre a incidência temporal de câncer cervical no Chile. Por outro lado, na Alemanha (base Saarland), apesar de não haver um programa de rastreamento instituído, as informações sobre a incidência estão disponíveis desde o início dos 1970, período em que há dados também para o Japão. Para países como Brasil, França, Itália, Holanda e Japão, há informações sobre incidência a partir dos anos de 1980, alguns coincidindo com o lançamento de programas nacionais de rastreamento. Observou-se que as taxas de incidência seguem em decréscimo para quase em todos os países, inclusive de modo bastante acentuado no Brasil e no Chile (Figura 2A). Observa-se ainda um aumento de incidência em países como Japão e Alemanha após o ano de 2008, cujas possíveis razões são discutidas na descrição de cada país realizada a seguir. É importante ressaltar que, excetuando Chile e Holanda que possuem valores globais, as taxas de incidência obtidas nos demais países são referentes a localizações ou regiões específicas selecionadas pela maior abrangência temporal na fonte de dados primários da Agência Internacional de Pesquisa em Câncer (IARC) da Organização Mundial da Saúde (WHO) [20].

Na Figura 2B é também mostrado o recorte temporal a partir de 1990, quando todos os países avaliados virtualmente utilizavam a citologia esfoliativa como método de diagnóstico, ainda que programas nacionais de rastreamento ainda não estivessem regularmente instituídos. Com exceção do Brasil, Chile e Alemanha, observou-se que as taxas permaneceram estáveis e abaixo de 10 casos/100 mil mulheres. Na Alemanha, que não possui programa de rastreamento instituído, as taxas sofrem variações, mas permanecem menores que 10 casos/100 mil mulheres após os anos 2000. No Brasil, onde há uma ampla variação entre as diferentes regiões, as taxas disponíveis e mostradas na Figura são referentes à Goiânia (uma das regiões com taxas mais altas do país). Outras regiões e estados apresentam taxas menores, porém não estão disponíveis na fonte de dados pesquisada. Portanto, para fins de padronização referencial, são mostrados os dados epidemiológicos constantes tão somente em Ferlay et al. (2018) [20]. Entretanto, os dados de outras regiões brasileiras poderão ser encontrados no sítio eletrônico do INCA (www.inca.gov.br) [1]. 
A

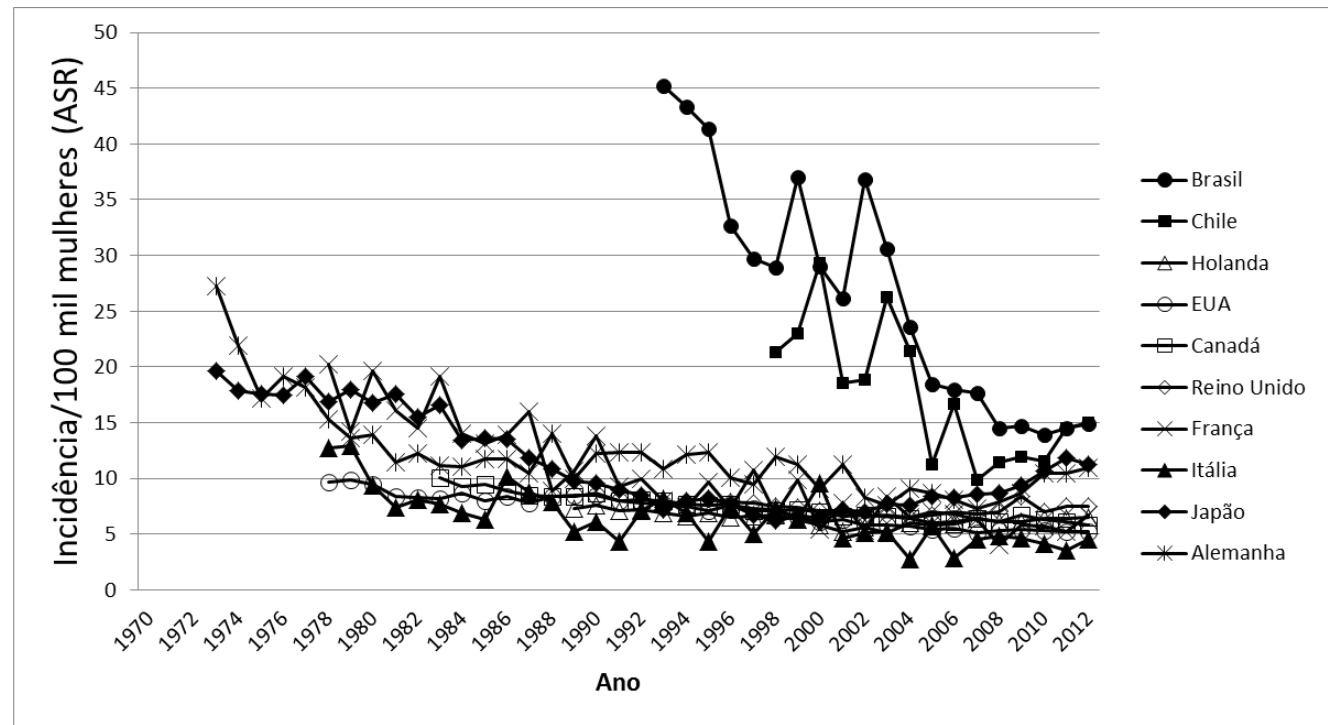

B

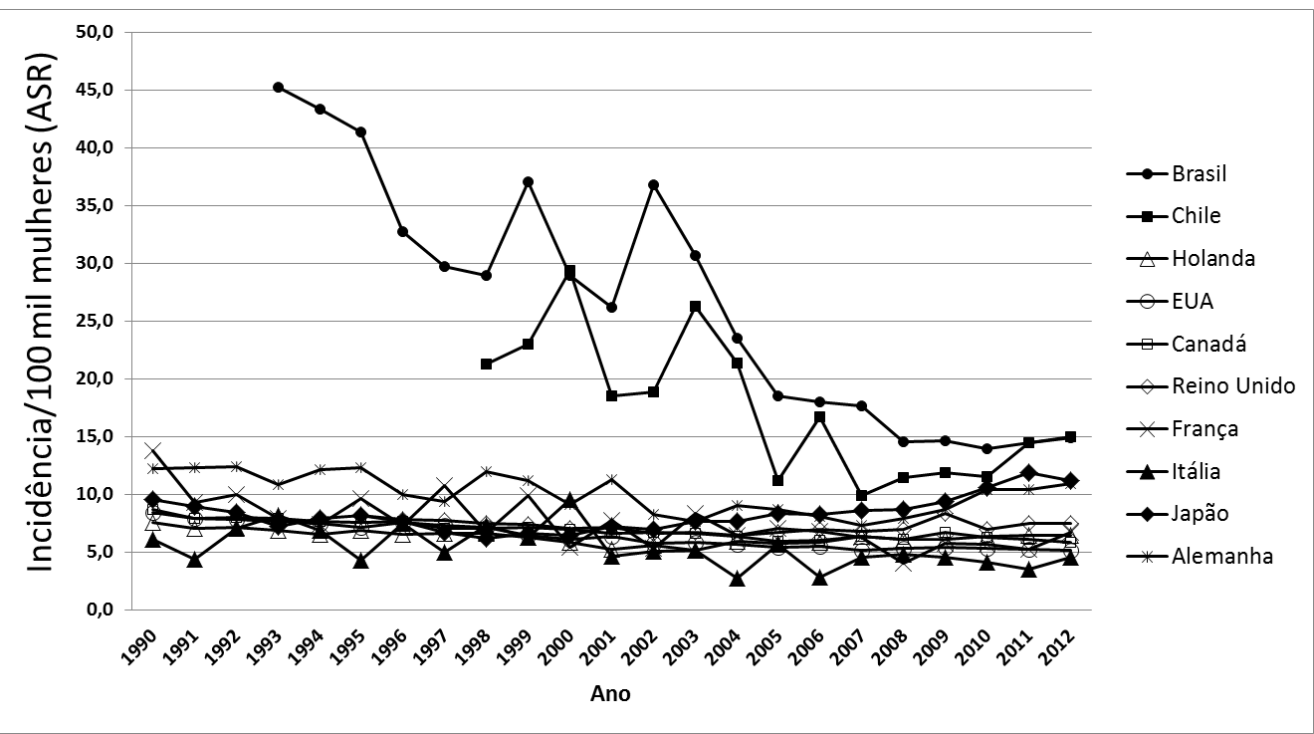

Figura 2. Evolução das taxas de incidência de câncer cervical nos dez países analisados entre os períodos de (A) 1970 a 2012 e (B) 1990 a 2012. Fonte primária dos dados: FERLAY, 2018 [20]. Disponível em < http://ci5.iarc.fr/CI5plus/Pages/online.aspx>. Acesso em 01abr2019. Referências específicas dos dados:

Brasil (Goiânia); EUA (SEER - The Surveillance, Epidemiology, and End Results Program); Japão (Osaka); Alemanha (Saarland); Itália (Parma); Reino Unido (Inglaterra); França (Calvados); Canadá (excluindo Nunavut, Quebec e Yukon).

\subsection{Programas de rastreamento populacional}

A Tabela 1 apresenta uma descrição sucinta da forma de organização dos programas de rastreamento nos diferentes países avaliados e suas principais características. Em seguida, são descritas de forma mais detalhada algumas características de seus diferentes programas de rastreamento. 
Tabela 1. Principais características dos programas de rastreamento implementados nos dez países selecionados para este estudo ${ }^{1}$.

\begin{tabular}{|c|c|c|c|c|c|c|}
\hline Países & Programa de rastreamento & Acesso da população & Idade & Intervalos de exame & Incidência/20126 & Incidência/2018 ${ }^{7}$ \\
\hline Brasil & Organizado/Oportunístico & Consulta aleatória & $25-64$ & 3 anos & 16,3 & 12,2 \\
\hline Chile & Organizado/Oportunístico & Consulta aleatória & $25-65$ & 3 anos & 12,8 & 12,2 \\
\hline Holanda & Organizado & Convite formal & $30-60$ & 5 anos (c/ teste HPV) & 6,8 & 5,7 \\
\hline EUA & Oportunístico & Consulta aleatória & $\begin{aligned} & 21-29 \\
> & 30 \text { anos }\end{aligned}$ & $\begin{array}{c}3 \text { anos } \\
5 \text { anos (c/ teste HPV) }\end{array}$ & 6,6 & 6,5 \\
\hline Canadá & Oportunístico & Consulta aleatória & $18(21)^{4}-69$ & $1-3$ anos & 6,3 & 5,7 \\
\hline Reino Unido & Organizado & Convite formal & $25-64$ & 3-5 anos & 7,1 & 8,4 \\
\hline França & Oportunístico/Organizado ${ }^{3}$ & Consulta aleatória/Convite 3 & $25-65$ & 3 anos & 6,8 & 6,7 \\
\hline Itália & Organizado/Oportunístico & $\begin{array}{l}\text { Consulta aleatória/Convite } \\
\text { formal }\end{array}$ & $25-64$ & 3 anos & 6,7 & 7,1 \\
\hline Japão & Organizado/Oportunístico & Consulta aleatória/Cupom ${ }^{2}$ & $>20$ anos & 2 anos & 10,9 & 14,7 \\
\hline Alemanha & $\begin{array}{c}\text { Não há programa oficial } \\
\text { implementado }\end{array}$ & Consulta aleatória & $>20$ & 1 ano & 8,2 & 7,5 \\
\hline
\end{tabular}

${ }^{1}$ Os dados apresentados na tabela foram compilados a partir das referências utilizadas no próprio texto.

${ }^{2}$ Em algumas províncias japonesas cupons para realização gratuita do exame de Papanicolaou são enviados às mulheres residentes (Ueda et al. 2015) [63].

${ }^{3}$ Algumas regiões têm implantado o programa de rastreamento organizado e há intenção de implantar nacionalmente o modelo organizado a partir de 2018 (Hamers et al. 2018) [52].

${ }^{4}$ Há diferenças nas faixas etárias nas diferentes províncias canadenses (PHAC 2009) [47].

${ }^{5}$ Será iniciado um programa piloto do tipo organizado em 2018 (von Karsa et al. 2015) [67]

${ }^{6}$ Taxa de incidência estimada e ajustada por idade (ASR) por 100 mil mulheres ano-base 2012 (Bruni et al. 2017) [69].

${ }^{7}$ Taxa de incidência estimada e ajustada por idade (ASR) por 100 mil mulheres ano-base 2018 (Ferlay et al. 2018) [21]. 


\subsection{Incidência nos países}

\subsubsection{Brasil}

O Brasil, teoricamente, possui um programa de rastreamento no modelo organizado [13], pois há uma população-alvo pré-definida, uma rede de atendimento para seguimento e acompanhamento com protocolos estabelecidos pelo Ministério da Saúde, e há um sistema de coleta de informações para obtenção dos indicadores epidemiológicos [14, 24], que preenchem os principais requisitos para um programa dito "organizado" [10]. A população alvo é de mulheres brasileiras na faixa etária de 25-64 anos [14] ou em qualquer faixa etária uma vez iniciada a vida sexualmente ativa [13], as quais deverão ser triadas/examinadas em intervalos trienais após dois testes negativos com intervalo anual ao ser admitida no programa. Entretanto, na prática, a população é rastreada no modelo oportunístico ou espontâneo, visto que não há um sistema de busca ativa da populaçãoalvo, tais como o envio de cartas-convite (recrutamento tipo call ou call/recall) ou algo dessa natureza. Essa sobreposição ou concomitância de características pertinentes aos programas organizados e oportunísticos ocorre em vários países, e quase sempre existe tendência em considerar como mais eficientes os programas estritamente organizados em detrimento dos estritamente oportunísticos [10,11, 25, 26]. Há ainda consenso sobre a importância de se manter os dois tipos de programas para fins de maior eficiência da ação [27].

No Brasil, regularmente, o exame de rastreamento é feito pela citologia convencional de Papanicolaou, ainda que a citologia líquida e testes moleculares para HPV possam ser solicitados a critério do médico responsável pela paciente. Todo o custo do programa de rastreamento, bem como o eventual tratamento e acompanhamento em casos de anormalidade é coberto pelo Sistema Único de Saúde (SUS). O programa de rastreamento brasileiro surgiu, inicialmente, por meio de um projeto-piloto criado no ano de 1996 e designado "Viva Mulher", dirigido a prevenção e detecção precoce do câncer cervical em mulheres com idade entre 35-49 anos nas seguintes localizações (Curitiba, Recife, Distrito Federal, Rio de Janeiro, Belém e Sergipe). Em 1998, o programa piloto foi expandido nacionalmente como Programa Nacional de Controle do Câncer do Colo do Útero - Viva Mulher [14]. Desde então diversas melhorias foram implantadas para aumentar a eficiência do programa, desde o controle da qualidade do exame laboratorial citológico por meio da Qualificação Nacional em Citopatologia (Qualicito) [28] até a criação de sistemas de gestão das informações, inicialmente denominado Sistema de Informação do Câncer do Colo do Útero (SisColo) e atualmente Sistema de Informações do Câncer (SisCan) [29].

Alguns estudos têm avaliado a eficiência do programa brasileiro em termos de cobertura da população-alvo. O estudo de Gakidou et al. (2008) [30] revelou que no Brasil a cobertura é de 73\%, variando de $60 \%$ a $90 \%$ entre a população mais pobre e a mais rica, respectivamente. Recentemente, o estudo de Barbosa (2017) [31] mostrou uma taxa de cobertura de 79,4\% com diferenças entre os Estados que variaram de 67,7 \% no Maranhão (mais baixa) a 86,5\% em Roraima (mais alta). É evidente que a taxa de cobertura tem melhorado nos últimos anos [32], mas ainda há diferenças expressivas entre as diferentes regiões que precisam ser corrigidas [31]. Essas diferenças regionais de cobertura podem refletir também em importantes diferenças regionais da incidência e mortalidade pelo câncer cervical [33].

Algumas sugestões de falhas no acompanhamento da mulher detectada com citologia anormal têm sido apontadas como fatores que contribuem para a não redução dos indicadores de incidência e mortalidade por câncer cervical no Brasil [34]. Evidentemente, não se consegue explicar de maneira simplista as razões para o Brasil apresentar a condição paradoxal de taxas de cobertura relativamente altas, próximas da taxa considerada ideal (>80\%) [35], quando comparadas aos países europeus, e ao mesmo tempo apresentar taxas altas de incidência e mortalidade. Certamente, mudanças pontuais na rede integrada do modelo organizado/oportunístico brasileiro para o câncer cervical ainda são necessárias [36]. 


\subsubsection{Chile}

Dentre os países selecionados, o Chile destaca-se por estar localizado na América Latina, por estar em desenvolvimento e por ter conseguido uma expressiva redução (aproximadamente 40\%) nas taxas de mortalidade por câncer cervical ao longo dos últimos anos (Figura 1B). Segundo o Ministério de Saúde Chileno (MINSAL), apesar de o Chile ofertar o exame de Papanicolaou para mulheres jovens numa abordagem aleatória e oportunística [37] desde 1965, o país deu início ao seu Programa Nacional de Pesquisa e Controle do Câncer Cérvico-Uterino somente no ano de 1987, concomitantemente a uma campanha de implementação e difusão orientada à realização do exame de Papanicolaou por mulheres de 25 a 64 anos. Destaca-se ainda que logo em seguida foi criado o Laboratório Nacional de Referência em Citologia Cervical, com implantação de um sistema de informatização de dados, publicação de material educativo e protocolos de atendimento baseados em medicina por evidência [38]. A partir de 2003, o Programa foi incorporado ao Programa de Garantias Explícitas de Saúde (GES-Chile) para assegurar a adequação de prazos máximos para diagnóstico, tratamento e seguimento dos casos. Em um balanço dos indicadores realizado em 2007, em evento de comemoração aos 20 anos de existência deste Programa no Chile, o MINSAL destacou: a cobertura do programa atingindo $66 \%$ das mulheres na faixa etária de 25-64 anos, o atendimento por especialista em até no máximo 30 dias para $98 \%$ das mulheres após encaminhamento pelo serviço primário e o início de tratamento em até 20 dias após confirmação de diagnóstico de câncer para $78 \%$ dos casos [38].

Desde então, o Chile vem atuando para aumentar a cobertura do seu programa de rastreamento, objetivando alcançar $80 \%$ das mulheres na faixa etária da população alvo, porém, a taxa de cobertura alcançada inicialmente sofreu redução para aproximadamente $60 \%$ em 2010 e vem permanecendo neste valor desde então [37]. Convém ressaltar que o câncer cervical no Chile é a segunda maior causa de mortes em mulheres de 15-44 anos no país, a sexta maior causa de mortes por câncer em geral, e as taxas de mortalidade ainda são mais que o dobro de países como os Estados Unidos da América [39, 40].

\subsubsection{Holanda}

A Holanda possui programa de rastreamento do câncer cervical na modalidade organizado em algumas cidades desde o ano de 1975, sendo reestruturado e tornado programa nacional no ano de 1996 [41]. Cartas-convite são enviadas pelas organizações de rastreamento ou pelos médicos da família (General practitioners) às mulheres pertencentes ao grupo populacional selecionado (faixa etária de 30-60 anos) para realização do exame em intervalos de 5 anos, nos casos de não haver anormalidade nos resultados. O exame é constituído pelo teste de Papanicolaou (citologia convencional ou LBC-Liquid Based Cytology) concomitantemente ao teste para detecção do HPV de alto risco (hrHPV). A combinação dos resultados determina o seguimento da paciente que poderá ser: retornar ao rastreamento intervalado de 5 anos, realizar nova citologia de acompanhamento em 6 meses ou ser encaminhada à colposcopia [41].

O Programa tem financiamento nacional pelo Ministério Holandês da Saúde, Bem-estar e Esportes e é organizado pelo Instituto Nacional Holandês de Saúde Pública e Ambiente (RIVM) [42]. $\mathrm{O}$ atendimento e o exame são realizados dentro da abrangência da atenção primária, pelo médico da família (General practitioners) e laboratórios conveniados, sem custo algum para a mulher. Porém, a paciente pode solicitar o exame diretamente nas consultas regulares com ginecologistas, neste caso, os custos ficam a cargo do seu seguro saúde. Na Holanda, o registro de indivíduos nas municipalidades é obrigatório, mesmo nas mudanças de província ou cidades. Esse registro, por estar sempre atualizado, é utilizado como base populacional para a seleção da população alvo e envio das cartas-convites. A cobertura do programa na Holanda é de 70\% [41].

Além de todo rastreamento ser bastante organizado, no sítio eletrônico do RIVM é possível obter informações completas sobre o programa: o modelo da carta-convite, a coleta do material, o modo como os resultados serão reportados. Além disso, há cartilhas informativas em diferentes línguas para o alcance das mulheres imigrantes que residem no país [42]. Nessa ordem de estruturação, a eficiência do programa holandês de rastreamento é confirmada por uma das menores taxas de 
incidência (6,8/100 mil mulheres em 2012) e mortalidade (1,41/100 mulheres em 2013) por câncer cervical do mundo [18].

\subsubsection{Estados Unidos da América}

Os EUA possuem programa de rastreamento para o câncer cervical no modelo oportunístico. Em razão de não haver um sistema americano universal e gratuito de saúde, a Lei 101-354 de 1990 instituiu o Breast and Cervical Cancer Mortality Prevention Act of 1990 criando então o National Breast and Cervical Cancer Early Detection Program (NBCCEDP). O NBCCEDP é gerido pelo Centers for Disease Control and Prevention (CDC) e objetiva ofertar os testes de rastreamento e detecção precoce às mulheres de baixa renda, ou seja, aquelas não seguradas e não atendidas pelos sistemas privados de saúde americanos [43]. Entretanto, a maior parte dos seguros de saúde nos EUA cobre os custos dos exames de rastreamento para o câncer cervical, tratamento e seguimento, funcionando adequadamente para as mulheres seguradas. Estima-se que mais que $90 \%$ dos americanos possuam seguro saúde [44], portanto, a cobertura ofertada pelo NBCCEDP é pequena quando comparada à população como um todo. Em 2000, a Lei 106-354 autorizou que mulheres detectadas com câncer na abrangência do NBCCEDP recebessem tratamento e seguimento pelos programas estaduais de saúde (MEDICAID) para pessoas de baixa renda [43].

O exame de rastreamento do câncer cervical está disponível em muitos serviços de saúde americanos: clínicas comunitárias e privadas, clínicas de doenças sexualmente transmissíveis, clínicas da família e de pré-natal [12]. A procura pelo serviço é totalmente espontânea, embora existam entidades sociais que estimulam e orientam as mulheres como obter o acesso ao programa NBCCEDP. Estima-se que 11,1\% das mulheres americanas sejam elegíveis ao programa e 6,5\% sejam efetivamente atendidas [43]. A organização American Cancer Society (ACS/USA) recomenda o uso da citologia convencional ou a LBC para mulheres na faixa etária entre 21-29 anos e o teste molecular para HPV somente para o acompanhamento dos casos com citologia alterada. Para mulheres entre 30-65 anos, recomenda-se o uso do co-teste (citologia esfoliativa e teste molecular de detecção do HPV) [45].

\subsubsection{Canadá}

No Canadá, o sistema de saúde é operado autonomamente pelas diferentes províncias, que seguem as recomendações das autoridades nacionais de saúde. O país possui sistema de saúde gratuito e universal (Fundo Universal de Saúde) com cobertura para os programas de rastreamento de doenças, incluindo a citologia para o câncer cervical, assim como o tratamento e seguimento dos casos.

O programa de rastreamento canadense para o câncer cervical segue majoritariamente o modelo oportunístico. Porém, há algumas províncias que implantaram modelos semi-organizados, chamados assim em razão da não existência de recrutamento sistematizado da população alvo [46], de modo similar ao existente no Brasil. O exame de rastreamento é realizado por citologia convencional de Papanicolaou ou LBC (dependendo da província) em todas as mulheres do grupo populacional (faixa etária de 18-69 anos, atualmente alterada para 21-69 anos). O intervalo entre exames é escalonado, um ano após o primeiro exame (com a idade de 18 ou 21 anos) e intervalos de três anos caso os dois primeiros testes tenham sido negativos [12, 47], similar ao modelo intervalar seguido no Brasil.

As taxas de coberturas são divulgadas como taxa de participação de mulheres na faixa etária alvo que fizeram ao menos um teste citológico no tempo da análise. Os últimos dados divulgados referem-se a 2010-2013 e mostraram uma taxa de participação ajustada por idade e histerectomia entre 64,9\% e 73,8\% para as províncias de Ontário e British Columbia, respectivamente, e entre $62,9 \%$ e $71,3 \%$ nas províncias de Saskatchewan e Newfoundland, respectivamente, não corrigidas para histerectomia. São taxas altas de participação para um país com modelo de rastreamento majoritariamente oportunístico e de procura espontânea pelo serviço. Um recente documento de atualização da associação The Canadian Partnership Against Cancer relatou que atualmente algumas províncias implantaram sistemas de carta-convite para recrutamento da população-base 
[48]. O recrutamento sistematizado por cartas-convites, possivelmente, impactará as taxas de participação no programa de rastreamento nos próximos anos.

\subsubsection{Reino Unido}

O Reino Unido adota o modelo de rastreamento populacional organizado com variadas formas de busca ativa da população alvo: envio de carta-convite, reenvio às não-atendentes (call/recall), telefonemas individuais para esclarecer dúvidas que estejam impedindo as mulheres de atender ao convite. O Programa Inglês existe desde 1988 com direção governamental (National Health Service - NHS/UK) incluindo ações de controle da qualidade desde 1995, um dos mais antigos dentre os países avaliados nesse estudo [12]. A população alvo inclui mulheres na faixa etária entre 25-64 anos que são formalmente convidadas a cada três anos (idade 25-49 anos) ou cinco anos (idade 5064 anos) pelo serviço de atenção primária onde a mulher está registrada, e em caso de não atendimento, o reenvio do convite ocorre após 18 semanas da primeira [49].

Uma característica de programa britânico de rastreamento é o uso exclusivo da LBC, ao invés da citologia convencional empregada prioritariamente em todos os demais países relatados. Além disso, o Reino Unido vem estudando há alguns anos a possibilidade de ampliar o uso de testes moleculares para HPV [12]. Outra característica importante é que o médico General practitioner é pago por número de pacientes triadas, uma medida que teve consequência no aumento da cobertura e no incentivo à realização do exame de rastreamento pelos médicos da atenção primária [12].

Ainda que possua um programa de rastreamento populacional organizado bem estabelecido, o Reino Unido (7,1/100 mil mulheres) ocupa a $12^{\mathrm{a}}$ posição entre países europeus com as mais baixas taxas de incidência de câncer cervical [50], situada atrás da Holanda (6,8/100 mil mulheres), França (6,8/100 mil mulheres) e Itália (6,7/100 mil mulheres). Para conhecimento, em 2012 a Suíça era o país com a mais baixa incidência (3,6/100 mil mulheres) de câncer cervical [18].

\subsubsection{França}

A França adota o modelo oportunístico para o rastreamento populacional do câncer cervical em praticamente todo o seu território desde o ano de 1960. Porém, algumas províncias implantaram o modelo organizado a partir do ano de 1990 [51], ainda que obrigatoriamente tenham que seguir as normas gerais do Ministério da Saúde Francês, tais como a população alvo (mulheres de 25-65 anos) e o intervalo entre os exames (3 anos). Segundo Schaffer et al. (2000) [51], até o final da década de 90, estimava-se que a França realizava 06 milhões de exames citológicos cervicais por ano, o suficiente para uma cobertura de $100 \%$ da população alvo. Entretanto, o autor ressalta que ainda assim $40 \%$ da população alvo relatavam nunca terem feito o exame de rastreamento, situação similar à da Itália, cujas causas são discutidas mais adiante [51].

Hamers et al. (2018) [52] relatam que, seguindo exemplos de outros países europeus, que indicam que os programas de rastreamento organizados com busca ativa são mais custo-eficientes na redução dos casos de câncer cervical, a França lançou estudos pilotos de implantação do modelo organizado em algumas regiões, com utilização estrita de carta-convite formal e personalizada, para avaliar a adequabilidade de uma ampliação para abrangência nacional [52]. Resultados preliminares foram divulgados constatando que houve um aumento de cobertura estimado em $12 \%$, o que segundo Hamers et al. (2018) [52] deve ser interpretado considerando as limitações do estudo: a real informação se o exame foi realizado por atendimento ao convite ou espontaneamente, a falta de grupo controle populacional de mulheres que não tenham recebido a carta-convite, o fato do exame não ter sido livre de custo para as mulheres em algumas cidades, o que pode ter reduzido a participação, entre outras. Ainda segundo os autores do estudo citado, o Ministério de Saúde Francês planeja o lançamento nacional em 2018 de um programa organizado totalmente gratuito a todas as mulheres convidadas. Objetiva-se reduzir a incidência do câncer cervical aumentando as taxas de cobertura que, atualmente, não ultrapassam os $60 \%$, principalmente nas mulheres acima de 40 anos [53]. 


\subsubsection{Itália}

Na Itália, o câncer cervical ocupa a $15^{\mathrm{a}}$ posição nos tipos de câncer de maior incidência em mulheres e a $3^{\mathrm{a}}$ posição entre mulheres de $15-44$ anos. Em mortalidade, o câncer cervical ocupa a $16^{\mathrm{a}}$ posição em mulheres em geral e a $7^{\mathrm{a}} \mathrm{em}$ mulheres na faixa etária de 15-44 anos, respectivamente [54]. A Itália possui um programa bem instituído de rastreamento organizado populacional, totalmente gratuito, que funciona concomitantemente às triagens oportunísticas (pública ou privada) que juntas conseguem alcançar cobertura de $79 \%$ da população alvo (mulheres de 25-64 anos). Dessas, $77 \%$ são regularmente convidadas (busca ativa) a participarem do programa de rastreamento [55]. O atendimento ao convite é variável dependendo da região do país, mas situase em torno de $40 \%$ em termos nacionais [56], indicando contribuição conjunta de ambos os métodos (organizado e oportunístico) para a alta cobertura.

O estudo de Rossi et al. (2009) [57] mostra uma característica interessante na Itália onde, virtualmente, haveria uma cobertura de $100 \%$ da população alvo, alcançado pelos dois modelos de triagem, porém, $30 \%$ das mulheres relatam não terem realizado triagem nos três anos anteriores, situação similar à existente na França. Os autores sugerem que isso ocorre porque há mulheres que realizam o exame em intervalos menores no triênio, enquanto outras ficam fora da cobertura, levando a uma análise quantitativa geral com cobertura virtual de $100 \%$. A realização do exame de Papanicolaou em intervalos menores do que os três anos preconizados é corroborada pelo estudo de Grazzini e Zappa (2008) [58] que mostrou que 34,2\% e 39,3\% das mulheres relataram ter realizado o exame com intervalos bienal e anual, respectivamente, enquanto apenas $13,7 \%$ haviam realizado no intervalo trienal regular.

Segundo Ronco et al. (2015) [59], a Itália, desde 2010, vem realizando estudos pilotos para avaliar a eficiência dos testes moleculares para detecção do HPV na prevenção das lesões cervicais ou mesmo no desenvolvimento do câncer cervical em nível populacional. Esses estudos subsidiarão as decisões governamentais sobre a implantação de testes moleculares no programa. Diversas regiões estão com estudos epidemiológicos em andamento e alguns resultados preliminares estão sendo publicados [59].

\subsubsection{Japão}

No Japão, o câncer cervical ocupa a décima posição entre os tipos de câncer com maior incidência (taxa bruta) em mulheres e a segunda maior causa de morte em mulheres na faixa etária de 15-44 anos [60]. Além disso, o Japão tem a maior taxa de incidência de câncer cervical (10,9/100 mil mulheres) entre os países do G7. Segundo Hamashima et al. (2010) [61], o Japão iniciou a oferta de rastreamento para o câncer cervical pelo uso do exame de Papanicolaou por volta de 1960 em cidades isoladas e somente em 1983, sob a coordenação do Health Service Law for the Aged, é que foi implantado o Programa Nacional de Rastreamento para todas as mulheres acima de 40 anos residentes no país [61]. Houve algumas mudanças no programa nos anos seguintes, tais como, recomendações de citologia convencional ou líquida, transferência do programa para gestões regionais com apoio do governo nacional e mudanças na faixa etária da população alvo que, atualmente, é constituída de mulheres com idade acima de 20 anos. Ainda assim, a cobertura do programa de rastreamento no Japão em 2006 era de apenas 18\% [61], a mais baixa entre os países selecionados neste estudo.

Ainda que existam programas institucionais não governamentais, tais como empresas e indústrias, que também ofertam às mulheres o serviço de rastreamento para câncer cervical como parte de exames de rotina, estima-se que o alcance da cobertura não supere os $25 \%$ [12]. Existem algumas hipóteses para essa baixa adesão das mulheres japonesas ao programa de rastreamento, uma delas é que há apenas uma cobertura parcial dos custos do exame, ficando o restante, entre 10$30 \%$ do valor, sob a responsabilidade de pagamento da mulher atendida [12]. Oshima e Maezawa (2013) [62] realizaram um estudo com mulheres jovens universitárias buscando explicar a baixa adesão desse grupo ao programa de rastreamento, a parte das limitações de alcance do estudo, os autores observaram que havia muita relutância para realização do exame motivada por: baixo incentivo para o exame, pouco conhecimento sobre o assunto e até mesmo algo como "ciclo de não realização do exame", por exemplo, algumas participantes relataram que por não conhecerem 
ninguém que tivesse realizado o exame anteriormente, também não se sentiam estimuladas a realizar. Convém relatar que a verificação da baixa cobertura que o programa japonês vinha apresentando fez com que algumas províncias, a partir de 2009, passassem a distribuir cupons às mulheres com idade entre 20 e 40 anos para realização do exame citológico gratuitamente, e ainda assim, a adesão vem se mantendo baixa [62,63].

\subsubsection{Alemanha}

A Alemanha destaca-se entre os países selecionados nesse estudo por não possuir um programa de rastreamento oficialmente instituído (governamental e nacional). Entretanto, o exame citológico de Papanicolaou está disponível gratuitamente (via reembolso governamental ou por seguro-saúde) para as mulheres acima de 20 anos (sem limite de idade) que procuram o serviço por demanda espontânea, podendo ser repetido anualmente [64]. Apesar de espontâneo, parece haver uma ampla conscientização da população alemã quanto à necessidade e disponibilidade dos testes no serviço de saúde alemão. Starker (2013) [65] avaliou a participação da população alemã em programas de rastreamento para câncer disponíveis no país: cervical, pele, próstata, mama e cólon, verificando que $88,5 \%$ das mulheres entrevistadas tinham conhecimento da recomendação e da necessidade de participar desses programas e 67,2\% das mulheres acima de 20 anos participavam regularmente de algum dos programas. Para o câncer cervical, as porcentagens de mulheres que relataram ter realizado o exame de Papanicolaou nos doze meses anteriores ao estudo foram de 52,8\% e 60,7\% para mulheres $>20$ anos e entre 40-49 anos, respectivamente. Para um país que não possui programa sistematizado de rastreamento, o alcance é bastante expressivo, possivelmente, atribuído ao nível educacional da população. Nesse contexto, Starker (2013) [65] ainda relata que mulheres com menor status socioeconômico informaram menores taxas de participação do que mulheres de maior status socioeconômico, variando em média de 33,6\% a 68,3\%, respectivamente. Outro estudo realizado por Geyer et al. (2015) [64], no estado de Niedersachsen, confirmou que a taxa de participação das mulheres em programas de rastreamento variou em média de 66,5\% e 67,6\% considerando os dois ou três anos anteriores, respectivamente. $\mathrm{O}$ autor relata ainda que há um pico de taxa de participação entre mulheres de 20-29 anos na Alemanha que decresce conforme aumenta a faixa etária.

Ainda que comprovada a eficiência da participação das mulheres nos programas de rastreamento para a redução das taxas de incidência e mortalidade por câncer cervical, as autoridades alemãs de saúde planejam reduzir ainda mais a incidência remanescente, pois se acredita ser decorrente da população que não participa do programa regularmente [66]. Sendo assim, o Federal Joint Committee G-BA, seguindo as recomendações da União Europeia quanto às medidas de prevenção do câncer cervical [67], autorizou que a partir do ano de 2018 seja instituído preliminarmente um programa de rastreamento organizado para o câncer cervical com envio de cartas-convite à população alvo (sistema de busca ativa). Portanto, mulheres de 20-60 anos receberão cartasconvites informando a necessidade de realização do exame citológico a cada 5 anos, e aquelas acima de 30 anos também realizarão o teste molecular para HPV. Segundo Hillemanns (2016) [68], esse programa piloto deverá ser analisado quanto à sua eficiência após alguns anos de implantação, buscando-se definir se o modelo é inferior ou superior ao modelo espontâneo, que também será mantido regularmente no país [68].

\section{CONSIDERAÇÕES FINAIS}

De modo geral, constatou-se que há casos de sucesso e insucesso para ambos os modelos de rastreamento empregados nos diferentes países. Simplificar as razões dos sucessos e insucessos por pontos estanques na tentativa de explicar o porquê de um país apresentar baixas incidência e mortalidade por câncer cervical associadas exclusivamente a determinado programa de rastreamento, não é adequada. Há os exemplos do EUA e Canadá que possuem programas oportunísticos e apresentam baixas taxas de incidência (o Canadá está em transição para um modelo organizado com busca ativa), e há casos de programas estritamente organizados, como no Reino Unido e Holanda, que apresentam sucesso traduzido em baixa incidência de câncer cervical. 
Portanto, outros fatores contribuem para o sucesso dos programas, que pode ser traduzido por uma maior adesão da população, aumento da cobertura e redução nas taxas de incidência e mortalidade. Fatores como nível educacional e socioeconômico, conscientização da oferta de rastreamento, crença na importância da detecção precoce e na prevenção da doença, custo ou gratuidade do exame para a paciente, dificuldade de acesso ao programa e até mesmo fatores de ordem psicológicas e emocionais têm sido avaliados e mostraram-se importantes, ainda que em diferentes graus, na tentativa de aumentar a eficiência dos programas. Esses fatores atuam em conjunto influindo concomitantemente na decisão da mulher em aderir ou não ao programa. Portanto, os gestores dos programas de rastreamento deverão estar aptos a diagnosticar o problema e atuar em diferentes frentes, com diferentes mecanismos, na tentativa de alcançar as mulheres não participantes. Programas de conscientização e educação em saúde certamente são bastante eficientes, mas os efeitos sobre os indicadores epidemiológicos tendem a aparecer em prazos maiores, característicos dos projetos educativos. Por outro lado, a busca ativa da população, a gratuidade e a facilidade de acesso ao programa parecem ter capacidade de produzir impactos mais diretos nas taxas de cobertura em curto prazo. Nesse sentido, Zeferino (2008) [34] no editorial ' $O$ desafio de reduzir a mortalidade por câncer do colo do útero' ressaltou uma característica importante que deve ser considerada pelos gestores de programas de rastreamento, especialmente, no caso do Brasil:

"Uma pessoa com uma dor intensa irá procurar os serviços de saúde independente das dificuldades de acesso, mas uma mulher assintomática não se submeterá às mesmas dificuldades para fazer um exame citológico preventivo" (Zeferino, 2008, pg. 214[34]).

Quanto à classificação dos programas de rastreamento: organizado ou oportunístico, há certa ambiguidade terminológica. Isso porque, considera-se 'organizado' aquele que possui uma rede sistematizada de atenção: população alvo definida por faixa etária e/ou gênero, diagnóstico, tratamento, seguimento dos casos, entre outros fatores. Entretanto, alguns programas oportunísticos também possuem rede sistematizada e, frequentemente, uma população alvo estabelecida. Nesse sentido, é mais eficiente diferenciá-los por: busca ativa da população alvo, intervalo entre os exames, controle do seguimento, encaminhamento e tempo de espera para consultar especialista, tratamento e ainda como as informações e dados são coletados e traduzidos em indicadores de eficiência. Isso porque, apesar do alcance da população alvo estar diretamente associado com as taxas de cobertura e, portanto, com a eficiência do programa, o impacto nos indicadores é dependente do funcionamento adequado de toda a cadeia de procedimentos da rede da atenção. Ou seja, um programa oportunístico que apresente todas as características de rede de atenção, mas que não esteja empenhado em aumentar a cobertura ou que não tenha o correto seguimento dos casos terá pouco efeito sobre as taxas de incidência e mortalidade. Isso parece ser o que ocorre no Brasil e, em certa medida, no Chile.

O Chile, apesar da expressiva redução das taxas de mortalidade e incidência do câncer cervical após implementação do programa nacional de rastreamento, não tem conseguido caminhar em direção à meta proposta de $80 \%$ de cobertura, portanto, o país precisa repensar as formas de aumentar a adesão populacional, assim como o Brasil. O Brasil, por outro lado, desde a implantação do seu programa nacional há quase vinte anos, não impactou as taxas de mortalidade e incidência do câncer cervical de forma expressiva, mesmo aumentando suas taxas de cobertura. Portanto, os gestores do programa de rastreamento do câncer cervical no Brasil devem realizar uma análise mais aprofundada para ajustar os pontos que não estão funcionando adequadamente no programa. $\mathrm{O}$ Japão, claramente, precisará trabalhar para aumentar a conscientização das mulheres quanto à importância de prevenir o câncer pelos métodos de rastreamento, aumentando as taxas de coberturas, ou mesmo estendendo a gratuidade. Tomando como modelos outros países como Alemanha, Canadá, França, e mesmo o Japão, que continuam buscando alternativas para aprimorar seus programas e impactar as taxas de incidência e mortalidade por câncer cervical, o Brasil deverá seguir caminho similar de autoavaliação e melhoria do seu programa.

Evidentemente, alguns destes países, mais avançados em termos de eficiência dos programas de rastreamento, poderão inclusive trabalhar com outras perspectivas de atuação, como a utilização de co-testes (citologia esfoliativa e teste molecular para HPV) ou focar suas ações em grupos 
específicos que, geralmente, apresentam pouca adesão aos programas, por exemplo: imigrantes, mulheres em faixas etárias mais altas ou aquelas economicamente mais vulneráreis. Essas ações mais específicas talvez preocupem o Brasil futuramente, pois atualmente, o foco deverá estar nas ações imediatas para continuidade da rede de atenção, ajustando as etapas da integralidade que não são adequadas e eficientemente cumpridas. O Brasil apresenta cobertura próxima a $80 \% \mathrm{em}$ algumas regiões e, paradoxalmente, apresenta incidência média de câncer cervical de 15 casos/100 mil mulheres. Outros países, com menor cobertura, apresentam taxas inferiores a 10 casos/100 mil mulheres. Portanto, no ajuste do programa brasileiro para impactar as taxas de incidência e mortalidade deverá ser considerada a heterogeneidade demográfica, social, educacional, econômica e mesmo geográfica existente no país.

\section{AGRADECIMENTOS}

À Kátia Yumi Uchimura, tutora do Curso de Especialização em Gestão de Redes de Atenção à Saúde da Escola Nacional de Saúde Pública Sérgio Arouca da Fundação Oswaldo Cruz (ENSP/FIOCRUZ), pelo acompanhamento na elaboração desse trabalho que foi apresentado como requisito obrigatório para conclusão do curso. À Escola Nacional de Saúde Pública Sérgio Arouca da Fundação Oswaldo Cruz pela oportunidade de formação proporcionada.

\section{REFERÊNCIAS BIBLIOGRÁFICAS}

1. INCA. Instituto Nacional de Câncer José Alencar Gomes da Silva. Atlas online de mortalidade do INCA, 2014. Disponível em <https://mortalidade.inca.gov.br/> Acesso em 15Jul2017.

2. CDC. 2014. U.S. Cancer Statistics Working Group. United States Cancer Statistics: 1999-2014 Incidence and Mortality Web-based Report. Atlanta: U.S. Department of Health and Human Services, Centers for Disease Control and Prevention and National Cancer Institute; 2017. Disponível em <www.cdc.gov/uscs>. Acesso em 16Jul2017.

3. Steiner E, Klubert D, Knutson D. Assessing breast cancer risk in women. Am Fam Physician. 2008; 78(12):1361-1366.

4. Worley S. Lung cancer research is taking on new challenges. Knowledge of tumors' molecular diversity is opening new pathways to treatment. Pharm \& Ther (P\&T). 2014;39(10):698-714.

5. Crosbie EJ, Einstein MH, Franceschi S, Kitchener HC. Human papillomavirus and cervical cancer. Lancet. 2013;382: 889-99.

6. Lopez MS, Baker ES, Maza M, Fontes-Cintra G, Lopez A, Carvajal JM, Nozar F, Fiol V, Schmeler KM. Cervical cancer prevention and treatment in Latin America. J Surg Oncol. 2017;9999:1-4. doi: $10.1002 /$ jso. 24544

7. WHO. Human papillomavirus (HPV) and cervical cancer. Fact sheet. Updated June 2016. Disponível em <http://www.who.int/mediacentre/factsheets/fs380/en/>. Acesso em 15Jul2017.

8. Bicca GLO, Silveira MF, Silva KRS, Barros FCLF. HPV infection and cervical cancer: a review of screening and preventive strategies in developed countries and brazilian policies. DST - J Bras Doenças Sex Transm. 2013;25(3):157-162. doi: 10.5533/DST-2177-8264-201325308

9. Hughes C. Cervical cancer: prevention, diagnosis, treatment and nursing care. Nurs Stand. 2009;23(27):4856. doi: $10.7748 / \mathrm{ns} 2009.03 .23 .27 .48 . c 6838$

10. Miles A, Cockburn J, Smith RA, Wardle J. A Perspective from Countries Using Organized Screening Programs. Cancer. 2004;101(5 Suppl):1201-13. doi: 10.1002/cncr.20505

11. Williams JH, Carter SM, Rychetnik L. 'Organised' cervical screening 45 years on: How consistent are organised screening practices? Eur J Cancer. 2014;50(17):3029-3038. doi: 10.1016/j.ejca.2014.09.005

12. IARC. Cervix cancer screening/IARC Working Group on the Evaluation of Cancer-Preventive Strategies (2004: Lyon, France). 2005.

13. INCA. Prevenção do câncer do colo do útero. Rev Bras Cancerol. 2003;49(4): 205.

14. INCA. Instituto Nacional de Câncer José Alencar Gomes da Silva. Coordenação de Prevenção e Vigilância. Divisão de Detecção Precoce e Apoio à Organização de Rede. Diretrizes brasileiras para o rastreamento do câncer do colo do útero. 2. ed. rev. atual. Rio de Janeiro: INCA, 2016.

15. CFR. Council on Foreign Relations. 2017. The Group of Seven (G7). Disponível em <https://www.cfr.org/backgrounder/group-seven-g7>. Acesso em 24Jul2017.

16. IMF. International Monetary Fund. 2017. Disponível em <http://www.imf.org/external/pubs/ft/weo/2016/02/weodata/weorept.aspx?pr.x=74\&pr.y=6\&sy=2015\& $\mathrm{ey}=2016 \&$ ssd $=1 \&$ sort $=$ country $\& d \mathrm{ds}=. \& \mathrm{br}=1 \& \mathrm{c}=213 \% 2 \mathrm{C} 218 \% 2 \mathrm{C} 223 \% 2 \mathrm{C} 228 \% 2 \mathrm{C} 233 \% 2 \mathrm{C} 288 \% 2 \mathrm{C} 29$ 
$3 \% 2 \mathrm{C} 248 \% 2 \mathrm{C} 366 \% 2 \mathrm{C} 336 \% 2 \mathrm{C} 298 \% 2 \mathrm{C} 299 \& \mathrm{~s}=\mathrm{NGDPDPC} \% 2 \mathrm{CPPPPC} \& \mathrm{grp}=0 \& \mathrm{a}=>$. Acesso em 24Jul2017.

17. NUMBEO. 2018. Disponível em <https://www.numbeo.com/quality-oflife/rankings_by_country.jsp?title=2018\&region=150>. Acesso em 19Dez2017.

18. Ferlay J, Soerjomataram I, Ervik M, Dikshit R, Eser S, Mathers C, Rebelo M, Parkin DM, Forman D, Bray, F. GLOBOCAN 2012 v1.0, Cancer Incidence and Mortality Worldwide: IARC CancerBase No. 11 [Internet]. Lyon, France: International Agency for Research on Cancer; 2013. Disponível em <http://globocan.iarc.fr>. Acesso em 31Ago2017.

19. WHO. World Health Organization, Department of Information, Evidence and Research, Mortality database. 2016. Disponível em <http://www-dep.iarc.fr/WHOdb/WHOdb.htm>. Acesso em 31Ago2017.

20. Ferlay J, Colombet M, Bray F. 2018. Cancer Incidence in Five Continents, CI5plus: IARC CancerBase No. 9 [Internet]. Lyon, France: International Agency for Research on Cancer; 2018. Disponível em: $<$ http://ci5.iarc.fr>. Acesso em 01abr2019.

21. Ferlay J, Ervik M, Lam F, Colombet M, Mery L, Piñeros M, Znaor A, Soerjomataram I, Bray F. 2018. Global Cancer Observatory: Cancer Today. Lyon, France: International Agency for Research on Cancer. Disponível em <https://gco.iarc.fr/today>. Acesso em 01abr2019.

22. Arrossi S, Sankaranarayanan R, Parkin DM. Incidence and mortality of cervical cancer in Latin America. Salud Publica Mex. 2003;45(suppl 3):S306-S314.

23. ICSN. The International Cancer Screening Network. Cervical Cancer Screening Programs in 19 ICSN Countries, 2012: Organization, Policies, and Program Reach. Disponível em <https://healthcaredelivery.cancer.gov/icsn/cervical/screening.html>, acessado em 31Ago2017.

24. INCA. Instituto Nacional de Câncer José Alencar Gomes da Silva. Coordenação de Prevenção e Vigilância. Estimativa 2016: incidência de câncer no Brasil. Rio de Janeiro: INCA, 2015.

25. Nieminen P, Kallio M, Anttila A, Hakama M. Organised Vs. Spontaneous Pap-Smear Screening for Cervical. Cancer: A Case-Control Study. Int J Cancer. 1999;83:55-58.

26. Adab O, McGhee SM, Yanova J, Wong CM, Hedley AJ. Effectiveness and Efficiency of Opportunistic Cervical Cancer Screening. Comparison with Organized Screening. Medical Care. 2004; 42(6):600-609. doi: 10.1097/01.mlr.0000128007.04494.29

27. Tranberg M, Larsen MB, Mikkelsen EM, Svanholm H, Andersen B. Impact of opportunistic testing in a systematic cervical cancer screening program: a nationwide registry study. BMC Public Health. 2015;15:681. doi: 10.1186/s12889-015-2039-0

28. BRASIL. Ministério da Saúde. Portaria no 3388, de 30 de dezembro de 2013. Brasília: Ministério da Saúde, 2013a.

29. BRASIL. Ministério da Saúde. Portaria no 3394, de 30 de dezembro de 2013. Brasília: Ministério da Saúde, 2013b.

30. Gakidou E, Nordhagen S, Obermeyer Z. Coverage of cervical cancer screening in 57 countries: Low average levels and large inequalities. PLoS Med, 2008; 5(6):e132. doi: 10.1371/journal.pmed.0050132

31. Barbosa IR. Regional and Socioeconomic Differences in the Coverage of the Papanicolau Test in Brazil: Data from the Brazilian Health Survey 2013. Rev Bras Ginecol Obstet. 2017;39(09): 480-487. doi: 10.1055/s-0037-1604481

32. Barcelos MRB, Lima RCD, Tomasi E, Nunes BP, Duro SMS, Facchini LA. Quality of cervical cancer screening in Brazil: external assessment of the PMAQ. Rev Saude Publica. 2017;51:67. doi: 10.1590/S1518-8787.2017051006802

33. Bruni L, Barrionuevo-Rosas L, Albero G, Serrano B, Mena M, Gómez D, Muñoz J, Bosch FX, de Sanjosé S. 2017b. ICO Information Centre on HPV and Cancer (HPV Information Centre). Human Papillomavirus and Related Diseases in Brazil. Summary Report 27 July 2017. Acesso em 24Set2017.

34. Zeferino LC. The challenge of reducing mortality due to cervical cancer. Rev Bras Ginecol Obstet. 2008;30(5):213-5. doi: 10.1590/S0100-72032008000500001

35. WHO. Cervical cancer screening in developing countries: report of a WHO consultation. 2002. Disponível em < http://apps.who.int/iris/bitstream/10665/42544/1/9241545720.pdf>. Acesso em 05Fev2018.

36. Derchain S, Teixeira JC, Zeferino LC. Organized, Population-based Cervical Cancer Screening Program: It Would Be a Good Time for Brazil Now. Rev Bras Ginecol Obstet. 2016;38:161-163. doi: 10.1055/s0036-1582399

37. Fica A. Prevención del cáncer cérvico-uterino en Chile: Mucha vacuna y poco Papanicolau. Rev Chil Infectol. 2014; 31(2):196-203. doi: 10.4067/S0716-10182014000200010

38. MINSAL. Ministério de Salud. Gobierno del Chile. 20 anos Programa de Cáncer cervicouterino. 2007. Disponível em < http://web.minsal.cl/portal/url/item/71716b3c3eed775ce04001011f0151ca.pdf>Acesso em 21 Set2017. 
39. Martelli Javiera L, Van De Wyngard V, Lagos M, Barriga MI, Puschel Illanes K, Ferreccio Readi C. Detección precoz del cáncer cervicouterino en Chile: tiempo para el cambio. Rev Méd Chile. 2014; 142(8):1047-1055. doi: 10.4067/S0034-98872014000800012

40. Bruni L, Barrionuevo-Rosas L, Albero G, Serrano B, Mena M, Gómez D, Muñoz J, Bosch FX, de Sanjosé S. 2017c. ICO Information Centre on HPV and Cancer (HPV Information Centre). Human Papillomavirus and Related Diseases in Chile. Summary Report 27 July 2017. Acesso em 24Set2017.

41. HCN. Health Council of the Netherlands. Population screening for cervical cancer. The Hague: Health Council of the Netherlands, 2011; publication no. 2011/07E.

42. RIVM. Nacional Institute for Public Health and the Environment. Cervical Cancer Screening Programme. Disponível em < http://www.rivm.nl/en/Topics/C/Cervical_cancer_screening_programme>. Acesso em 23 Set 2017.

43. CDC. Center for Disease Control and Prevention. Updated 2017. National Breast and Cervical Cancer Early Detection Program (NBCCEDP). Disponível em https://www.cdc.gov/cancer/nbccedp/about.htm>. Acesso em 23Set2017.

44. Barnett JC, Berchick ER. Current Population Reports, P60-260, Health Insurance Coverage in the United States: 2016, U.S. Government Printing Office, Washington, DC, 2017.

45. ACS. American Cancer Society. The American Cancer Society Guidelines for the Prevention and Early Detection of Cervical Cancer. Updated 2016. Disponível em < https://www.cancer.org/cancer/cervicalcancer/prevention-and-early-detection/cervical-cancer-screening-guidelines.html>. Acesso em 23 Set2017.

46. CPAC. Canadian Partnership Against Cancer. Cervical Cancer Screening in Canada: Monitoring \& Evaluation of Quality Indicators. Toronto: Canadian Partnership Against Cancer. Updated July 2016. Disponível em

https://content.cancerview.ca/download/cv/prevention_and_screening/cccic_microsite/documents/cccic monitoringevalqualityindicatorspdf $>$. Acesso em 23Set2017.

47. PHAC. Public Health Agency of Canada. Performance Monitoring for Cervical Cancer Screening Programs in Canada. 2009. Disponível em < https://www.canada.ca/content/dam/phacaspc/migration/phac-aspc/cd-mc/cancer/pmecspc-srpdccuc/pdf/cervical-eng.pdf $>$. Acesso em 23 Set2017.

48. CPAC. Canadian Partnership Against Cancer. Cervical Cancer Screening in Canada: Environmental Scan [Internet]. Toronto (ON): Canadian Partnership Against Cancer; 2017. Disponível em <http://content.cancerview.ca/download/cv/prevention_and_screening/screening_and_early_diagnosis/d ocuments/cervicalcancerenviroscanpptx>. Acesso em 23Set2017.

49. NHS/UK. National Health Service, United Kington. Guidance Cervical screening: programme overview. Disponível em <https://www.gov.uk/guidance/cervical-screening-programme-overview>. Acesso em 22 set2017.

50. CANCER RESEARCH UK. Cervical Cancer Statistics. Disponível em $<$ http://www.cancerresearchuk.org/health-professional/cancer-statistics/statistics-by-cancertype/cervical-cancer\#heading-Zero>. Acesso em 22 Set 2017.

51. Schaffer P, Sancho-Garnier H, Fender M, Dellenbach P, Carbillet JP, Monnet E, Gauthier GP, Garnier A. Cervical cancer screening in France. Eur J Cancer. 2000:36:2215-2220. doi: 10.1016/S09598049(00)00312-9

52. Hamers FF, Duport N, Beltzer N. Population-based organized cervical cancer screening pilot program in France. Eur J Cancer Prev. 2018 Sep;27(5):486-492. doi: 10.1097/CEJ.0000000000000365

53. INCa/Fr. Les cancers en France en 2016 - L'essentiel des faits et chiffres. Collection Les Données / Epidémiologie. Février 2017. Disponível em <http://www.e-cancer.fr/Expertises-etpublications/Catalogue-des-publications/Les-cancers-en-France-en-2016-L-essentiel-des-faits-etchiffres>. Acesso em 22Set2017.

54. Bruni L, Barrionuevo-Rosas L, Albero G, Serrano B, Mena M, Gómez D, Muñoz J, Bosch FX, de Sanjosé S. 2017e. ICO Information Centre on HPV and Cancer (HPV Information Centre). Human Papillomavirus and Related Diseases in Italy. Summary Report 27 July 2017. Acesso em 22Set2017.

55. Campari C, Fedato C, Iossa A, Petrelli A, Zorzi M, Anghinoni E, Bietta C, Brachini A, Brezzi S, Cogo C, Giordano L, Giorgi D, Palazzi M, Petrella M, Schivardim MR, Visiolie CB, Rossi PG. Cervical cancer screening in immigrant women in Italy: a survey on participation, cytology and histology results. Eur $\mathbf{J}$ Cancer Prev. 2016; 25:321-328. doi: 10.1097/CEJ.0000000000000173

56. Ronco G, Giubilato P, Naldoni C, Zorzi M, Anghinoni E, Scalisi A, Palma PD, Zanier L, Barca A, Angeloni C, Gaimo MD, Maglietta R, Mancini E, Pizzuti R, Iossa A, Segnan N, Zappa M. Extension of organised cervical cancer screening programmes in Italy and their process indicators, 2010 activity. Epidemiol Prev. 2012;36(6) Suppl.1: 1-96. 
57. Rossi PG, Ricciardi A, Cohet C, Palazzo F, Furnari G, Valle S, Largeron N, Federici A. Epidemiology and costs of cervical cancer screening and cervical dysplasia in Italy. BMC Public Health. 2009;9:71. DOI: $10.1186 / 1471-2458-9-71$

58. Grazzini G, Zappa M. Attendance in cancer screening programmes in Italy. Italian J Pub Health. 2008; 5(2):117-124. doi: $10.2427 / 5839$

59. Ronco G, Giorgi-Rossi P, Giubilato P, Del Mistro AR, Zappa M, Carozzi F. A first survey of HPV-based screening in routine cervical cancer screening in Italy. Epidemiol Prev. 2015;39(3) S1: 77-83.

60. Bruni L, Barrionuevo-Rosas L, Albero G, Serrano B, Mena M, Gómez D, Muñoz J, Bosch FX, de Sanjosé S. 2017d. ICO Information Centre on HPV and Cancer (HPV Information Centre). Human Papillomavirus and Related Diseases in Japan. Summary Report 27 July 2017. Acesso em 21Set2017.

61. Hamashima C, Aoki D, Miyagi E, Saito E, Nakayama T, Sagawa M, Saito H, Sobue T. The Japanese Guideline for Cervical Cancer Screening. Jpn J Clin Oncol. 2010;40(6)485-502. doi: 10.1093/jjco/hyq036

62. Oshima S, Maezawa M. Perception of Cervical Cancer Screening Among Japanese University Students Who Have Never Had a Pap Smear: A Qualitative Study. Asian Pac J Cancer Prev. 2013;14(7):43134318. doi: 10.7314/APJCP.2013.14.7.4313

63. Ueda Y, Sobue T, Morimoto A, Egawa-Takata T, Hashizume C, Kishida H, Okamoto S, Yoshino K, Fujita M, Enomoto T, Tomine Y, Fukuyoshi J, Kimura T. Evaluation of a Free-Coupon Program for Cervical Cancer Screening Among the Young: A Nationally Funded Program Conducted by a Local Government in Japan. J Epidemiol. 2015;25(1):50-56. doi: 10.2188/jea.JE20140080

64. Geyer S, Jaunzeme J, Hillemanns P. Cervical cancer screening in Germany: group-specific participation rates in the state of Niedersachsen (Lower Saxony). A study with health insurance data. Arch Gynecol Obstet. 2015;291:623-629. doi: 10.1007/s00404-014-3421-3

65. Starker A. Participation in cancer screening programmes. Results of the German Health Interview and Examination Survey for Adults (DEGS1). Bundesgesundheitsbl, 2013;56:858-867. DOI: 10.1007/s00103-012-1655-4.

66. Schneider V. Cervical cancer screening in Germany. Current status. Pathologe. 2012;33(4):286-92. doi: 10.1007/s00292-012-1579-7

67. von Karsa L, Arbyn A, De Vuyst H, Dillner J, Dillner L, Franceschi S, Patnick J, Ronco G, Segnan N, Suonio E, Törnberg S, Anttila A. 2015. Executive summary. In: European guidelines for quality assurance in cervical cancer screening. Second edition, Supplements. Anttila A, Arbyn A, De Vuyst H, Dillner J, Dillner L, Franceschi S, Patnick J, Ronco G, Segnan N, Suonio E, Törnberg S \& von Karsa L (eds.). Office for Official Publications of the European Union, Luxembourg, pp. XIII-XXIV.

68. Hillemanns P. The paradigm shift in cervical cancer screening in Germany. Arch Gynecol Obstet. 2016; 293(1):3-4. doi: 10.1007/s00404-015-3940-6

69. Bruni L, Barrionuevo-Rosas L, Albero G, Serrano B, Mena M, Gómez D, Muñoz J, Bosch FX, de Sanjosé S. ICO Information Centre on HPV and Cancer (HPV Information Centre). Human Papillomavirus and Related Diseases in the World. Summary Report 27 July 2017. Acesso em 22Set2017. 